\title{
Teriparatide as option for severe osteoporosis
}

\author{
Mara CARSOTE ${ }^{1,2}$, Adina GHEMIGIAN ${ }^{1,2}$, Otilia RADU², Ana VALEA ${ }^{3}$ \\ 1"C.I. Parhon" National Institute of Endocrinology \\ 2"Carol Davila" University of Medicine and Pharmacy, Bucharest \\ ${ }^{3}$ Clinical County Hospital, "I. Hatieganu” University of Medicine and Pharmacy, Cluj-Napoca
}

\section{ABSTRACT}

We introduce an original study referring to Romanian population treated with teriparatide (TPT), an anabolic drug for severe menopausal, glucocorticoid and male hypogonadism-related osteoporosis. Primary end point is to analyze the parameters of persons who fulfilled the national criteria of TPT regarding co-morbidities and bone profile. Secondary end point is to reveal the skeleton indices 12 months after TPT (20 $\mu \mathrm{g} /$ day subcutaneous). Informed written consent was signed between July 2015 and June 2016. Out of 21 patients with a mean age of 66.76 years (yrs), except for 2 men, there were menopausal females with av. 21.47 yrs since menopause. $57 \%$ had a history of superior digestive condition, another $57 \%$ had a chronic thyroid disease and $29 \%$ had a non-thyroid autoimmune morbidity. 17 patients were pre-exposed to anti-osteoporotic drugs 4.23+/-3.49 yrs. Number of prevalent fractures was: 3.75+/-2.17 (median 3; min 1, max 9). 42\% (N=9) of subjects were followed for 1 year: no new fracture was registered; each patient had at least one DXA site with a BMD (Bone Mineral Density) increase (mean T-score increase of the most affected region was of 0.56+/-0.2 $S D$ ); osteocalcin statistically significant elevated from $18.87+/ 4.22 \mathrm{ng} / \mathrm{mL}$ to $42.8+/-16.3 \mathrm{ng} / \mathrm{mL}(p<0.0005)$ while CrossLaps went from $0.46+/-0.22 \mathrm{ng} / \mathrm{mL}$ to $0.65+/-0.3 \mathrm{ng} / \mathrm{mL}(\mathrm{p}=0.15)$. Early drop-offs were registered in 2 patients.

Based on this original study, patients with severe osteoporosis having a burden of many years of prior therapy or fragility fractures became candidates for TPT. After 12 months, the anabolic window was revealed by high bone remodelling blood markers, especially for osteocalcin.
\end{abstract}

Keywords: teriparatide, osteoporosis, fragility fracture

\section{INTRODUCTION}

Osteoporosis, especially the menopausal type, has become a medical and economical burden despite recent discoveries and implemented protocols of screening and therapy (1-3). The treatment varies from anti-resorbtives as bisphosphonates and denosumab to bone forming agents as teriparatide (TPT, the sequences 1-34 of human parathormone), approved by FDA in 2002 (1-3). TPT has also been studied in European clinical trials, as EUROFORS, and currently it is accessible in Romania through a specific protocol of inclusion, exclusion and prescription, referring for the entire country (daily subcutaneous injections of $20 \mu \mathrm{g}$ for 24 months) (4).

\section{OBJECTIVE}

We introduce an original study referring to Romanian population treated with TPT. Primary end point is to analyze the parameters of persons who fulfilled the criteria of TPT access regarding co-morbidities and bone profile. Secondary end point is to reveal the skeleton indices 12 months after TPT was started.

\section{MATERIAL AND METHOD}

The design of the study is observational, descriptive with a cross-sectional part, while a subset of subjects was also included into a longitudinal study (a retrospective analysis after

\section{Correspondence:}

Mara Carsote MD, PhD, "C.I. Parhon" National Institute of Endocrinology, 34-38 Aviatorilor Ave, District 1, Bucharest, Romania

E-mail: carsote_m@hotmail.com 
12 months since TPT was initiated). TPT was prescribed in $20 \mu \mathrm{g}$ per day via self-administrated injections. The longest period of time an osteoporotic subject may be treated with TPT is two years, once in a lifetime, without drop offs and resumptions. The inclusion criteria are according to the national recommendations: men and women with severe menopausal, glucocorticoid, and hypogonadism-related male osteoporosis who were previously treated or not with bisphosphonates. The patients with prior specific anti-osteoporotic medication experienced side effects or were non-responders based on loosing Bone Mineral Density (BMD) or having a new fragility fracture or continuing to have low T-score after 5 years of bisphosphonates. The exclusion protocol criteria were hypercalcemia, hyperparathyroidism, primary or secondary bone malignancies, radiotherapy, Paget's disease. The parameters included data provided by anamnesis and personal patients' records (as prior fractures, treatment history, medical and surgical conditions prior to admission). Body Mass Index (BMI) was calculated $(\mathrm{kg} / \mathrm{sqm})$. The blood tests were done at baseline: total and ionic calcium, phosphorus, 25-hydroxyvitamin D (25-OH D), parathormone (PTH), bone formation markers (osteocalcin, alkaline phoaspatase and, also, P1NP which was off-protocol), and bone resorption marker CrossLaps. Apart from national requirement, we provided 24-hour urinary calcium measurement. Central DXA (Dual-Energy X-Ray Absorptiometry) was done at least one site (lumbar, femoral neck, total hip) through a GE Lunar Prodigy machine. Profile X-Ray at the lumbar and thoracic level was performed at baseline and after one year. Out of national protocol, all the women had a bilateral mammography done at baseline or within the last six months before inclusion (as well as Pap test). After one year of daily TPT, the bone parameters, DXA, spine X-ray were repeated. In the meantime, the subjects were offered vitamin $D$ and calcium supplements based on individual decision. We used Excel database; descriptive parameters were based on Mean (av.), Median, SD (Standard Deviation), minimum (Min), maximum (Max); statistical significance was at $p<0.05$. Each patient signed an informed consent regarding the use of their medical records and assays either when they were just enrolled or during follow-up, between July 2015 and June 2016. This is an experience of a single first-prescriber of TPT from a tertiary Romanian centre of endocrinology regardless the fact the patients were followed by different endocrinologists before and after TPT was first administered. The evaluation before one year was also done by the same first prescriber. In cases of drop-offs, a supplementary assessment was performed also at the tertiary endocrinology centre to confirm the reasons of stopping TPT.

\section{RESULTS}

Out of 21 included patients, two were men (representing 9.5\%). The entire cohort had a mean age of 66.76 years (yrs) (Table 1). The females were all in menopause and had an average period of time of $21.47+/-11.73$ yrs since menopause (median of $21 \mathrm{yrs}$ ). Mean BMI was $23.66+/-4.73 \mathrm{~kg} / \mathrm{sqm}$ (median of $24 \mathrm{~kg} / \mathrm{sqm}$ ). The percent of current smokers was $9.5 \%$; also $9.5 \%$ of subjects were under corticotherapy (more than daily $5 \mathrm{mg}$ of prednisone or equivalent for at least three months); $14.28 \%$ of patients were obese (with a BMI of at least $30 \mathrm{~kg}$ / sqm). $57.14 \%$ of the studied population had a medical history of a superior digestive condition as ulcer or oesophagitis, which did not allowed oral therapy with bisphosphonates. The co-morbidities included: $57 \%$ of patients with a thyroid chronic disease $(\mathrm{N}=12$, six of them had multi-nodular goitre, three had hypothyroidism, two had treated hyperthyroidism and one had chronic thyroiditis); $29 \%(\mathrm{~N}=6)$ of all had a non-thyroid autoimmune chronic morbidity (two with ankylosing spondilytis, one with scleroderma, one with thrombocytopenia, one with asthma, and one with psoriasis) (Fig. 1). The baseline bone blood and urinary bone indices are displayed in Table 2 and 3. The inclusion criteria of each patient were classified as "non-responder DXA" meaning an $8 \%$ loss of BMD at central DXA per year of non-TPT anti-osteoporotic drug or a T-score of $\leq-3$ SD after 5 years or oral/injectable bisphosphonates and "non-responder fracture", meaning the confirmation of a fragility fracture while the patient was treated with bisphosphonates. The distribution of

TABLE 1. Age distribution at baseline when the TPT (teriparatide) was first administered after adequate evaluation according to inclusion criteria of Romanian protocol ( $N=21$ subjects)

\begin{tabular}{|l|l|}
\hline \multicolumn{2}{|c|}{ Age at starting TPT (years) } \\
\hline mean & 66.76 \\
\hline median & 64 \\
\hline stdev. & 8.94 \\
\hline minimum & 47 \\
\hline maximum & 83 \\
\hline
\end{tabular}


TABLE 2. Baseline bone profile before therapy with teriparatide was initiated; *25-OH D (25-hydroxyvitamin D) was first adjusted to be at least $30 \mathrm{ng} / \mathrm{mL}$ before first injection); $P T H=$ parathormone

\begin{tabular}{|l|l|l|l|l|l|l|}
\hline & $\begin{array}{l}\text { Total calcium } \\
(\mathbf{m g} / \mathbf{d l})\end{array}$ & $\begin{array}{l}\text { lonic calcium } \\
\text { (mg/dL) }\end{array}$ & $\begin{array}{l}\text { Urinary calcium } \\
\text { (g/24-h) }\end{array}$ & Phosphorus $(\mathbf{m g} / \mathbf{d L})$ & 25-OH D (ng/mL) & PTH (pg/mL) \\
\hline mean & 9.47 & 4.09 & 0.14 & 3.72 & $29.6^{*}$ & 40.262 \\
\hline median & 9.4 & 4.11 & 0.11 & 3.9 & $27.4^{*}$ & 41.12 \\
\hline stdev. & 0.4 & 0.24 & 0.1 & 0.72 & $13.37^{*}$ & 9.17 \\
\hline normal & $8.5-10.2$ & $3.9-4.9$ & $0.07-0.3$ & $2.5-4.5$ & $30-100$ & $15-65$ \\
\hline
\end{tabular}

TABLE 3. Bone remodelling markers at baseline (just before therapy with teriparatide was started)

\begin{tabular}{|l|l|l|l|l|}
\hline Baseline & $\begin{array}{l}\text { Alkaline } \\
\text { Phosphates } \\
(\mathbf{U} / \mathrm{L})\end{array}$ & $\begin{array}{l}\text { P1NP } \\
(\mathrm{ng} / \mathrm{mL})\end{array}$ & $\begin{array}{l}\text { Osteocalcin } \\
(\mathrm{ng} / \mathrm{mL})\end{array}$ & $\begin{array}{l}\text { CrossLaps } \\
(\mathrm{ng} / \mathrm{mL})\end{array}$ \\
\hline mean & 72.23 & 46.40 & 16.39 & 0.32 \\
\hline median & 66 & 30.54 & 16.19 & 0.26 \\
\hline stdev. & 27.79 & 38.56 & 4.66 & 0.19 \\
\hline normal & $38-105$ & $15-74$ & $15-46$ & $0.226-1.008$ \\
\hline
\end{tabular}

TABLE 4. Central DXA assessment when teriparatide was started $(N=21)$ according to each region (GE Lunar Prodigy device)

\begin{tabular}{|l|l|l|l|}
\hline & $\begin{array}{l}\text { Lumbar } \\
\text { T-score }\end{array}$ & $\begin{array}{l}\text { Femoral } \\
\text { neck T-score }\end{array}$ & $\begin{array}{l}\text { Total hip } \\
\text { T-score }\end{array}$ \\
\hline mean & -3.3 & -2.5 & -2.28 \\
\hline median & -3.3 & -2.4 & -2.2 \\
\hline stdev. & 0.67 & 0.85 & 1.05 \\
\hline non-useful data (no./\%) & $4(19 \%)$ & $2(9.5 \%)$ \\
\hline
\end{tabular}

TABLE 5. Changes of BMD (Bone Mineral Density) according to central DXA scan in patients who were treated with teriparatide $20 \mu \mathrm{g} /$ day for a year $(N=9)$

\begin{tabular}{|l|l|l|l|l|l|l|}
\hline DXA & $\begin{array}{l}\text { Lumbar BMD: } \\
\text { baseline }\end{array}$ & $\begin{array}{l}\text { Lumbar BMD: } \\
\mathbf{1 2} \text { months }\end{array}$ & $\begin{array}{l}\text { Femoral neck } \\
\text { BMD: baseline }\end{array}$ & $\begin{array}{l}\text { Femoral neck } \\
\text { BMD: 12 } \\
\text { months }\end{array}$ & $\begin{array}{l}\text { Total hip BMD: } \\
\text { baseline }\end{array}$ & $\begin{array}{l}\text { Total hip BMD: } \\
\mathbf{1 2} \text { months }\end{array}$ \\
\hline mean & 0.792 & 0.861 & 0.702 & 0.760 & 0.734 & 0.758 \\
\hline median & 0.795 & 0.873 & 0.729 & 0.765 & 0.735 & 0.781 \\
\hline stdev. & 0.065 & 0.087 & 0.117 & 0.109 & 0.161 & 0.166 \\
\hline$p$ value & 0.09 & 0.3 & & 0.7 & \\
\hline
\end{tabular}

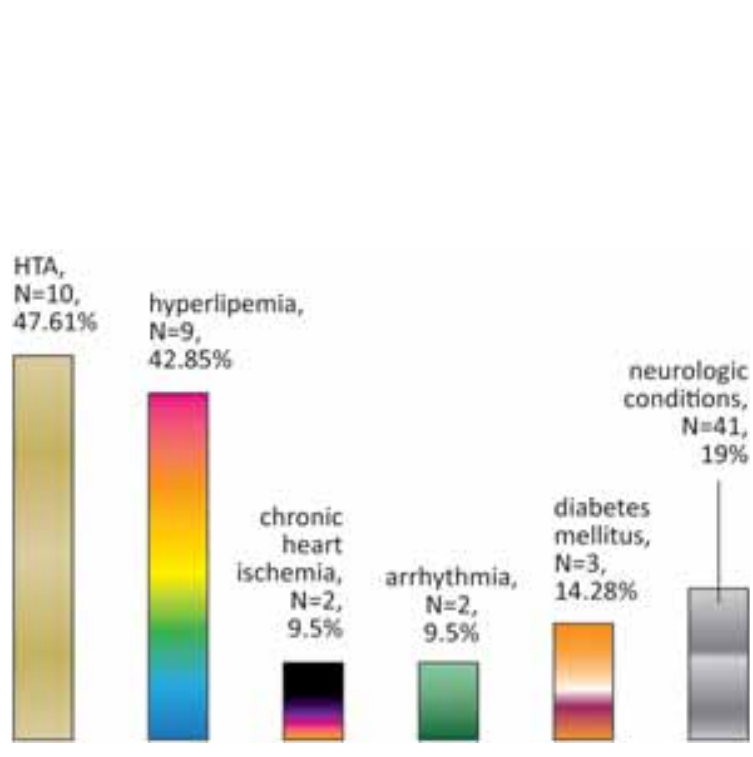

FIGURE 1. The cardiovascular and neurologic conditions of the enrolled patients treated with teriparatide $(N=21)$

subjects according to these adapted criteria is displayed in Fig. 2. Seventeen patients had a history of different medication for osteoporosis until TPT was initiated (vitamin D and calcium are not included) (Fig. 3). The prior anti-osteoporotic drug time was 4.23+/-3.49 yrs (median $3 \mathrm{yrs}$; min 0; max $14 \mathrm{yrs}$ ). The number of prior fractures was $3.75+/-2.17$ (median 3 ; min 1,

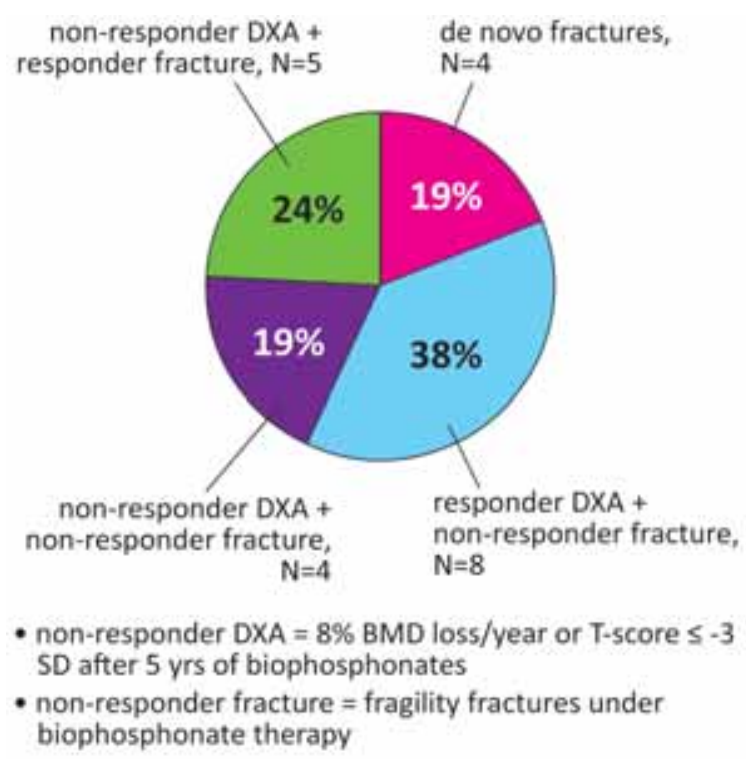

FIGURE 2. The distribution of patients treated with TPT according to inclusion criteria (referring to DXA assays and prior osteoporotic fractures with or without previous specific medication for osteoporosis)

$\max 9)$. Only $14 \%$ of patients did not have vertebral fractures $(\mathrm{N}=3)$. Each vertebra and rib was considered separately if the confirmation of the fracture (of different severity) was done. Apart from spine X-Ray, it was necessary, for the positive and differential diagnosis, to perform whole body bone scintigram and/or computer tomography of different sites in one third of cases with 

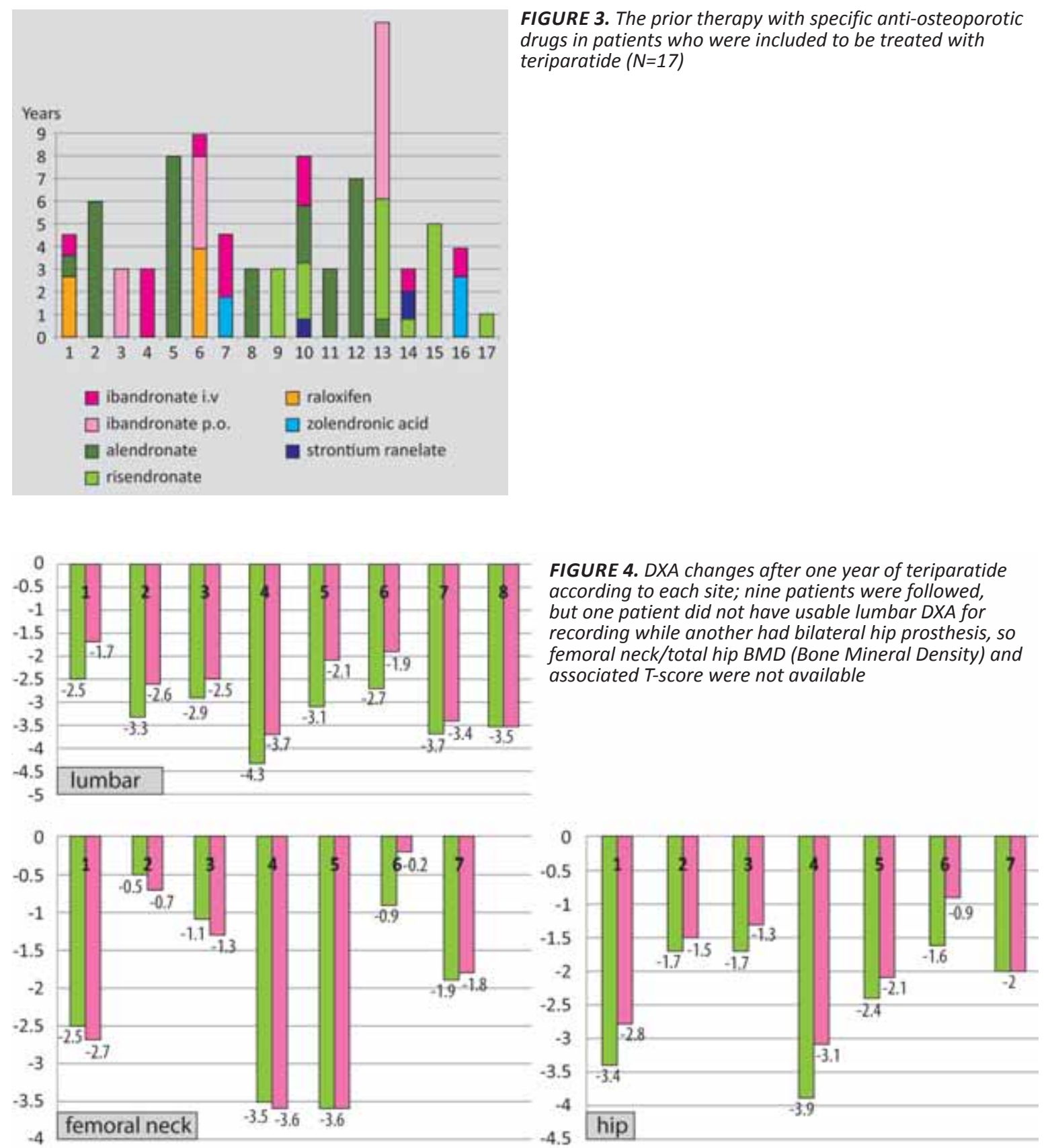

FIGURE 3. The prior therapy with specific anti-osteoporotic drugs in patients who were included to be treated with teriparatide $(N=17)$

FIGURE 4. DXA changes after one year of teriparatide according to each site; nine patients were followed, but one patient did not have usable lumbar DXA for recording while another had bilateral hip prosthesis, so femoral neck/total hip BMD (Bone Mineral Density) and associated T-score were not available an individual decision. All the patients had at least one central DXA site with a T-score of $\leq$ -2.5 , and up to one fifth of subjects had at least one non-interpretable DXA situs due to fracture damage or severe coxarthrosis (Table 4). After one year, the re-analysis was done for 9 (42\%) subjects. No new fracture was registered and each patient had at least one DXA site with a BMD increase. The mean increase of T-score regarding the most affected region was of $0.56+/-$ $0.2 S D$ (median of $0.6 \mathrm{SD}$ ). For these subjects, BMD changes did not reach statistically significance except for a tendency for lumbar spine (Table 5, Fig. 4). Bone remodelling markers were initially very high or suppressed if the patients were pre-treated (Fig. 5). After 12 months of daily TPT, osteocalcin statistically significant increased from $18.87+/ 4.22 \mathrm{ng} / \mathrm{mL}$ (median of 16 ng.mL) to $42.8+/-16.3 \mathrm{ng} / \mathrm{mL}$ (median of 40.87 $\mathrm{ng} / \mathrm{mL}$ ), with $\mathrm{p}<0.0005$ but not CrossLaps from $0.46+/-0.22 \mathrm{ng} / \mathrm{mL}$ (median of $0.22 \mathrm{ng} / \mathrm{mL}$ ) to $0.65+/-0.3 \mathrm{ng} / \mathrm{mL}$ (median $0.3 \mathrm{ng} / \mathrm{mL}$ ) with $p=0.15$ (Fig. 6). Early drop-offs were registered in two patients: one female of 83 years who accused non-specific heart beat complains after 3 months of TPT remitted when TPT was stopped and one male of 63 years who complained of vertigo, dizziness, nausea and muscle pain (after 2 months of TPT) which stopped when TPT was no longer administered. 

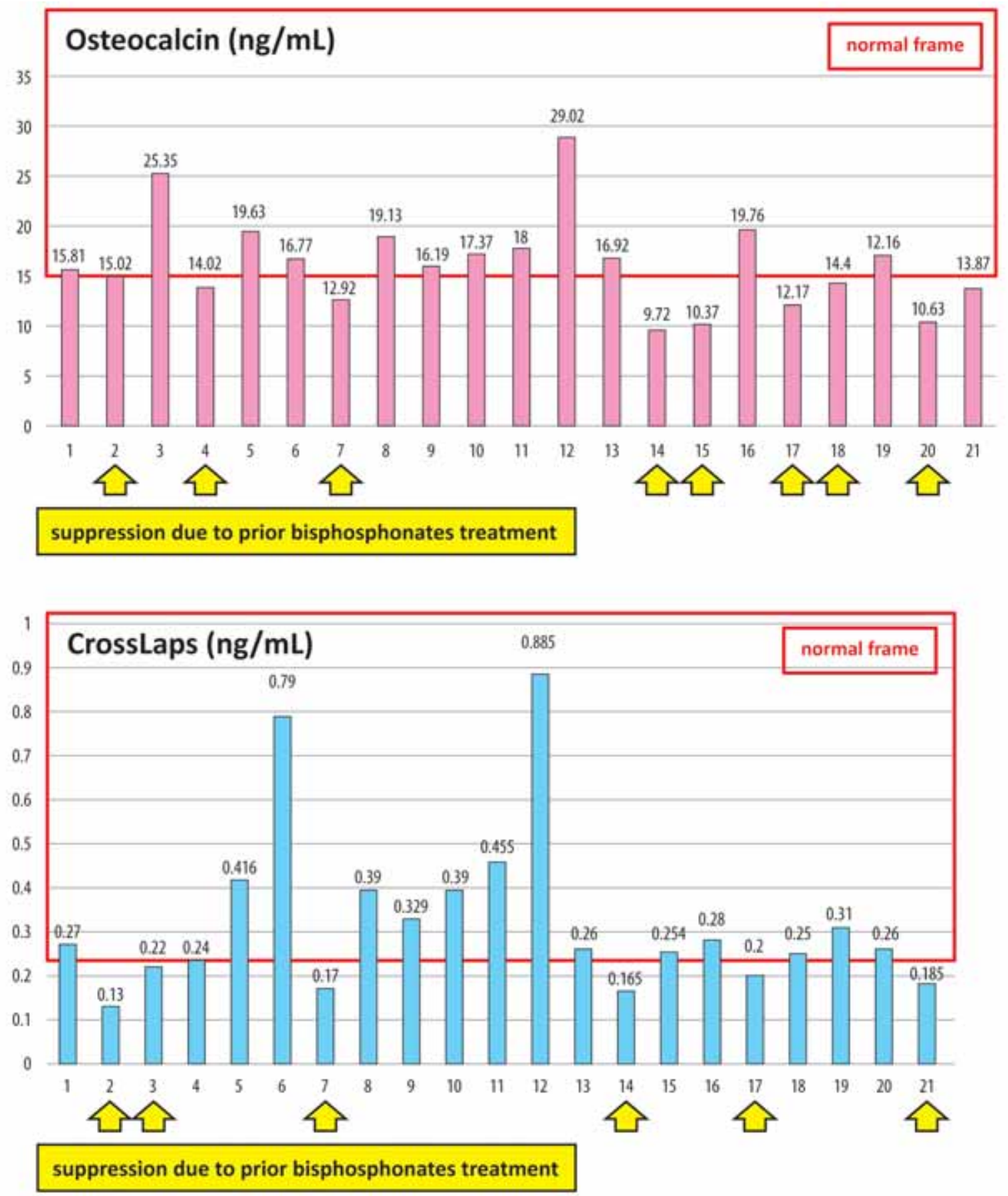

FIGURE 5. The bone remodeling markers at the beging of teriparatide therapy: osteocalcin as bone formation and Crosslaps as bone resorption; red frame represents the normal interval; yellow arrow represents supresed values on some of the patients priorly treated with anti-resorptives $(N=21)$

\section{DISCUSSION}

To our knowledge, this study is one of the first of its kind referring to real life medicine for Romanian population in accordance to criteria of TPT prescription that were introduced almost two years ago in current form and are restricted to endocrinologists. The limits of the study are small sample size, single first-centre experience, the difficulty of interpreting the drop-offs, the need for completing the 24 months protocol. However, overall, a population with a long medical history of anti-osteoporotic drugs, multiple fractures and severe T-score became candidate for TPT. Moreover, a consistent number of patients had non-interpretable DXA sites due to co-morbidities. As non-bone pathology, we mention upper digestive chronic diseases that interfere with oral drugs as bisphosphonates. The fact than more than one half of the patients 

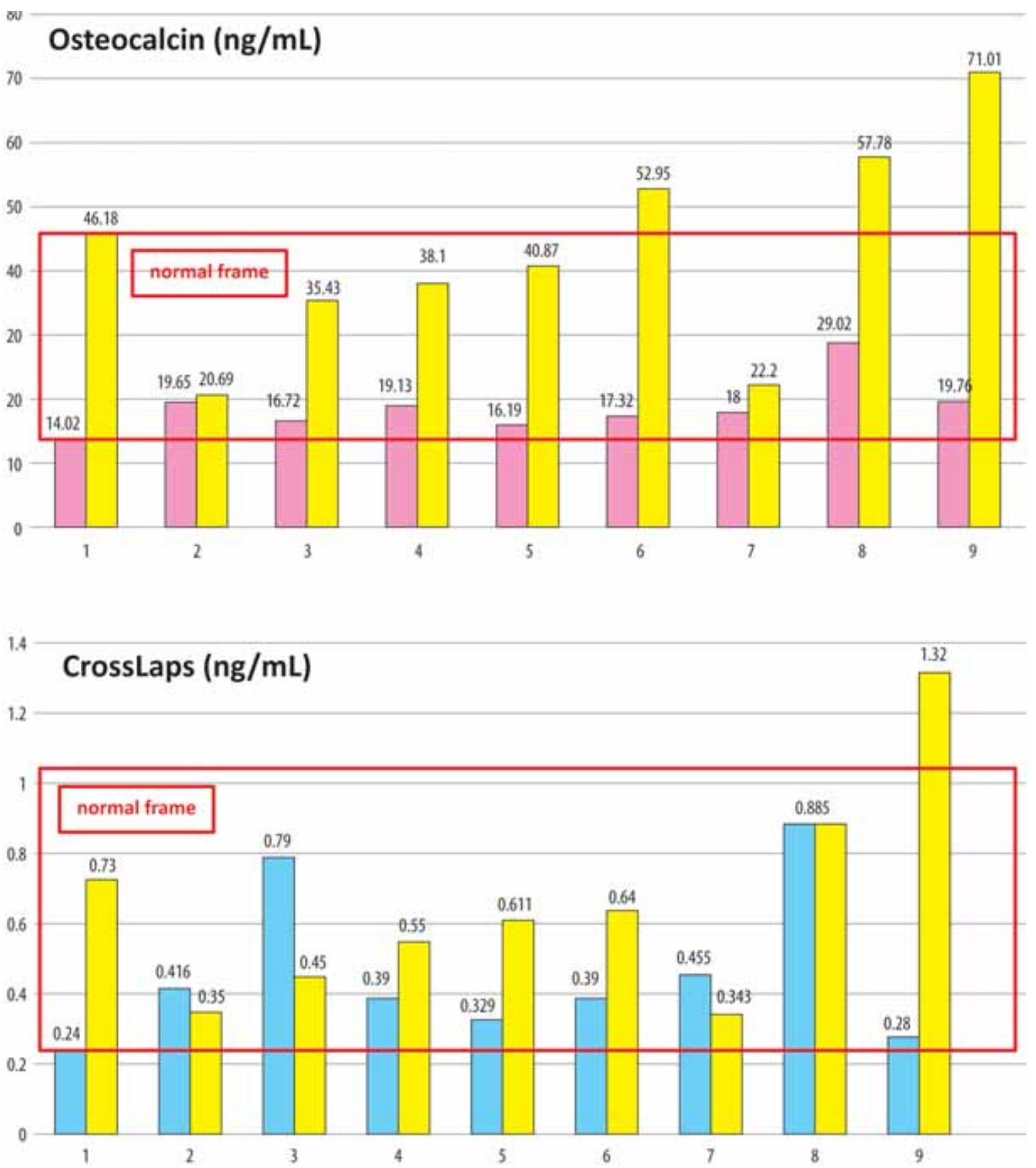

FIGURE 6. The bone turnover markers of each patient treated with teriparatide $20 \mu \mathrm{g}$ per day, 12 months; red frame represents the normal interval $(N=9)$

had either abnormal thyroid ultrasound or TSH at some moment is related mostly to routine endocrine assays for a tertiary centre rather than skeleton anomalies background. The analysed data after one year of TPT pointed a BMD improvement (each patient became its self control) and the most interesting aspect is reaching the anabolic window due to osteocalcin values (5).

\section{CONCLUSION}

Based on our observations, patients with severe osteoporosis having a burden of many years of prior therapy or fragility fractures became candidates for teriparatide. After one year, no new fracture was seen, with a BMD increase and a statistical significant bone formation osteocalcin elevation, accordant to anabolic action.

Acknowledgement: We thank the patients and our co-workers who helped up with patients' selection and investigations.

Conflict of interest: The study is independent of any bias, material benefits or pharmaceutical company. MC has been a speaker for Eli Lilly Romania within the last 12 months. 


\section{REFERENCES}

1. Drake M.T., Clarke B.L., Lewiecki E.M. The Pathophysiology and Treatment of Osteoporosis. Clin Ther. 2015 Aug; 37(8): 1837-50.

2. Andreopoulou P., Bockman R.S. Management of postmenopausal osteoporosis. Annu Rev Med. 2015; 66:329-42.
3. Maeda S.S., Lazaretti-Castro M. An overview on the treatment of postmenopausal osteoporosis. Arq Bras Endocrinol Metabol. 2014 Mar; 58(2): 162-71.

4. Graeff C., Timm W., Nickelsen T.N. et al. Monitoring teriparatide-associated changes in vertebral microstructure by high-resolution $\mathrm{CT}$ in vivo: results from the EUROFORS study. J Bone Miner Res. 2007 Sep; 22(9):1426-33.

5. Bilezikian J.P. Anabolic therapy for osteoporosis. Womens Health (Lond). 2007 Mar; 3(2):243-53. 